\title{
Scrofula: emergency department presentation and characteristics
}

\author{
Nicolas Forget • Kathryn Challoner
}

Received: 18 January 2009 /Accepted: 13 June 2009/Published online: 18 August 2009

(C) Springer-Verlag London Ltd 2009

\begin{abstract}
Background In the US, scrofula is generally uncommon, but it may be the manifestation of disseminated tuberculosis in immunocompromised patients. Given the delay to obtain PPD results, AFB results, and cultures for TB, the emergency physician (EP) must rely on the history and physical examination to make the diagnosis of scrofula.

Aims To illustrate a set of criteria that would be useful to the emergency physician to identify cases of scrofula. Methods We retrospectively reviewed the charts of patients with a final diagnosis of scrofula at our institution to identify the characteristics of patients who present to the emergency department with a neck mass that was eventually diagnosed as scrofula.

Results We found that being foreign born, being HIV+, and having a prior history of a positive PPD appeared to be associated with a diagnosis of scrofula.

Conclusions This review suggests that scrofula should be included in the EP's differential diagnosis of neck in masses when patients present subacutely and they have significant TB risk factors. In such cases, the EP should strongly consider ruling out pulmonary TB.
\end{abstract}

Keywords Scrofula Extra pulmonary tuberculosis . Emergency department . Cervical tuberculous lymphadenopathy $\cdot$ Mycobacterium tuberculosis

The views expressed in this paper are those of the author(s) and not those of the editors, editorial board or publisher.

N. Forget $(\triangle) \cdot K$. Challoner

Division of International Emergency Medicine,

Department of Emergency Medicine, Keck School of Medicine,

University of Southern California,

1200 N State St, Room 1011,

Los Angeles, CA 90033, USA

e-mail: forget@usc.edu

\section{Introduction}

In 2007, the United States recorded over 13,000 cases of tuberculosis (TB) [1], mainly in large cities with significant immigrant populations such as New York, San Francisco, and Los Angeles [2]. Cervical tuberculous lymphadenitis or scrofula is the most common form of extra-pulmonary TB and is the presenting form of the disease in $5 \%$ of the cases. However, in immunocompromised patients, scrofula may be the presenting form of TB in a third of cases [3].

In adults, $95 \%$ of cases are caused by Mycobacterium tuberculosis and are best treated with pharmacologic therapy. In contrast, in pediatric cases, $92 \%$ of lesions are caused by non-tuberculous mycobacterium and respond best to surgical treatment.

Although scrofula is generally rare, as emergency physicians (EP) working with indigent, immigrant, and immunocompromised patients, it is a condition that we may encounter and should be able to recognize. Missing a diagnosis of scrofula is indeed a missed opportunity to diagnose a patient with pulmonary TB. Without the luxury of purified protein derivative (PPD) results, acid-fast bacilli (AFB) results, and culture results for TB, we must rely mainly on the history and physical examination to make the diagnosis. It is important to distinguish scrofula from an abscess or other mass as scrofula may hide a transmittable form of the disease. The authors present a case series of scrofula at a large urban county hospital with a significant immigrant and indigent population in an attempt to highlight the clinical features that will assist the emergency physician in making this important diagnosis.

\section{Materials and methods}

After obtaining IRB approval, we retrospectively reviewed all charts with a discharge diagnosis of scrofula among 
patients seen at our institution in the past 10 years. Nineteen separate patients were identified. Complete charts were available for eight cases. The other charts were not retrievable from the archives. We had preselected data to be extracted based on prior publications [4-7]. These data points were patient demographics, their comorbidities, the details of their presentation, the diagnostic studies, and finally the treatment. All personal identifiers were removed and stored securely and separately from the database. Given the small number of available charts, we present the results in the form of a case series.

\section{Cases}

Patient 1 was a 43-year-old female born in Cambodia who had moved to the US 5 years prior to presentation. She reported having an enlarging left-sided neck mass for the past 3 months. She had initially been seen at our institution 3 months prior for the same complaint. Her fine-needle aspiration (FNA) at the time was negative for AFB. Nevertheless, she was started on isoniazid, rifampin, pyrazinamide, and ethambutol (RHZE). She admitted to weight loss and night sweats. On physical examination she had an $8 \times 6-\mathrm{cm}$, tender, fluctuant mass with erythematous borders on her left neck. She was admitted to the hospital. Her chest X-ray was negative, and her sputum AFBs were negative. A repeat FNA of her abscess eventually showed $2+$ AFB. The patient was compliant with her medications and her condition resolved (Table 1).

Comment: This case illustrates a few significant risk factors: the patient is from a TB-endemic country with a history of a positive PPD. She does have constitutional symptoms, but does not have pulmonary TB as illustrated by her negative chest X-ray, sputums for AFB, and culture.

Patient 2 was a 33-year-old Korean man with no past medical history who presented to the ED with a right axillary mass growing for the past 1 month. He denied any fevers, chills, or night sweats. His physical examination showed three mobile masses with the largest being $3 \times$ $3.5 \mathrm{~cm}$ at the right axilla. No erythema or warmth was noted. He was admitted to the hospital where an FNA of his mass was performed. The smear was negative for AFB, but his culture grew out Mycobacterium tuberculosis. His sputum was negative for AFB, but he did have a positive

Table 1 Summary of cases

\begin{tabular}{|c|c|c|c|c|c|c|c|c|}
\hline Patient & 1 & 2 & 3 & 4 & 5 & 6 & 7 & 8 \\
\hline Age & 43 & 33 & 60 & 32 & 57 & 55 & 38 & 43 \\
\hline Sex & $\mathrm{F}$ & M & M & M & $\mathrm{F}$ & $\mathrm{F}$ & M & M \\
\hline Country of birth & Camb & Kor & USA & Mex & Kor & USA & USA & USA \\
\hline Years in US & 5 & NA & 60 & 12 & 18 & 55 & 38 & 43 \\
\hline HIV & - & - & + & + & - & - & + & + \\
\hline Other TB RF & None & None & Jail, TB & None & None & Homeless & TB & Homeless \\
\hline PPD & + & - & NA & + & NA & + & + & NA \\
\hline CXR & - & - & + & + & - & - & + & + \\
\hline Sputum AFB & - & - & + & - & - & - & - & + \\
\hline Sputum culture & NA & - & NA & + & - & NA & - & + \\
\hline Location & Neck & Axilla & Neck & Neck & Neck & Neck & Neck & Neck \\
\hline Duration of mass (wk) & 12 & 4 & 12 & 3 & 3 & 4 & 2 & NA \\
\hline FNA AFB & $-/ 2+$ & - & NA & $2+$ & $3+$ & $1+$ & $3+$ & $3+$ \\
\hline FNA culture & NA & + & NA & - & - & NA & - & + \\
\hline Treatment & RHZE, L, S + I\&D & RHZE & RHZE + I\&D & RHZE & RHZE & RHZE & RHZE & RHZE \\
\hline
\end{tabular}

Camb: Cambodia

Kor: Korea

Mex: Mexico

NA: Data not available

TB RF: Other TB risk factors

L: Levofloxacin

S: Streptomycin

CXR: Chest X-ray, + indicates non-normal. See cases for complete results

RHZE: Rifampin, isoniazid, pyrazinamide, and ethambutol 
PPD. He was treated with the standard four-drug regimen (RHZE).

Comment: This case illustrate how tricky the diagnosis may be as the patient's only risk factor is being from an TB-endemic country. In addition, the emergency physician is more likely to incise and drain a mass in the axilla, thus placing the patient at higher risk for prolonged drainage. His lesion did have the appearance of a "cold abscess" (no erythema or warmth), which is more typical of scrofula.

Patient 3 was a 60-year-old African-American male who presented to the ED with a left-sided neck mass for the past 3 months. He had a prior history of HIV, hepatitis B and C, and tuberculosis. His social history was significant for incarceration and intravenous drug use. He was already on TB treatment at presentation. On examination, he had a $6 \times$ $7-\mathrm{cm}$, tender mass in the submandibular area of the left neck. It was erythematous and fluctuant, but with no active drainage. He was admitted to the hospital. His workup included a negative chest X-ray, a CD4 count of 205, and one sputum positive for AFB. The ENT service performed an open incision and drainage (I\&D) with washout of his mass. He was discharged on RHZE, but died a year later of unclear causes.

Comment: this case illustrates that immunocompromised patients are at higher risk of having disseminated TB, or at least a transmittable form of the disease, thus making their admission to respiratory isolation paramount. His case also illustrates how present TB can be in certain segments of the US-born population, such as incarcerated and homeless persons.

Patient 4 was a 32-year-old male born in Mexico and living in the US for the past 12 years who presented to the ED with a 3-week history of progressively enlarging masses on his left neck. They were erythematous and without drainage. The patient denied fevers, chills, shortness of breath, weight loss, or night sweats. He reported a non-productive cough and rhinorrhea. He denied intravenous drug use and was homosexual in a monogamous relationship. His physical examination showed a $2 \times 2-\mathrm{cm}$, slightly indurated, mobile and mildly tender mass over the medial left clavicle. The second lesion was $3 \times 3-\mathrm{cm}$ along the medial aspect of the sternocleidomastoid border. His chest X-ray showed a right upper lobe opacity. His workup revealed a positive HIV serology with a CD4 count of 147, a positive $\mathrm{PPD}$, a sputum positive for $\mathrm{AFB}$, and a positive sputum culture. FNAs of his neck masses were $2+$ AFB. He was started on RHZE prior to discharge and followed up at an outside clinic.

Comment: This case presents a confluence of circumstances that will likely be more common in the future: the patient was born in an TB-endemic country and is HIV positive. In addition he was unaware of his HIV-positive status prior to presentation, another common scenario in the
ED. He was immunocompromised, had disseminated TB, and was thus contagious.

Patient 5 was a 57 -year-old Korean female who had been living in the US for 18 years. She presented to the ED with a right neck mass for the past 10 years that had increased in size for the past 3 weeks. She acknowledged a 15- to 20pound weight loss in the past 3 months. She also reported that her husband had been diagnosed with pulmonary TB 16 years ago. On examination, she had a $1.5 \times 1.5-\mathrm{cm}$ neck mass in the right posterior cervical area. It was tender, erythematous, and had been draining since an FNA the week prior. Her chest X-ray was negative. Her FNA was positive for $\mathrm{AFB}$, so she was placed in respiratory isolation, and RHZE was initiated from the ED. Her sputums were AFB and culture negative. She successfully completed 9 months of RHZE treatment with no documented recurrences.

Comment: This case represents a classic case of transmission among close contacts and illustrates the need for prompt evaluation for pulmonary TB to prevent further transmission.

Patient 6 was a 55-year-old African-American female who presented to the ED with a left-sided neck mass for 1 month. She also had a cough. She reported a history of syphilis and a repeatedly positive PPD in the years prior to presentation. Her chest X-ray was negative, and she had three negative sputums for AFB. She was discharged from the ED, and her scheduled FNA was AFB positive, but culture negative. The patient was started on RHZE.

Comment: This case is the only one in our series of a US-born person who remains HIV negative. She did have a positive PPD in the past and a history of homelessness, which should alert the EP to her risk of scrofula and possibly disseminated disease.

Patient 7 was a 38-year-old African-American male who presented to the ED with bilateral neck masses for the past 2 weeks. He reported a mild, dry cough, a 20-pound weight loss in the past month, subjective fevers, but no hemoptysis and no night sweats. The patient was HIV positive, on antiretrovirals, and had a history of a negative PPD a year prior to presentation. On examination, he had a left-sided, slightly tender, $7 \times 5-\mathrm{cm}$ mass with no warmth or erythema. On the right, he had a $4 \times 2-\mathrm{cm}$ mass. A CT scan of the neck showed bilateral lymphadenopathy with fluid collection. The patient was started on antibiotics for a suspected neck abscess and was admitted. His chest X-ray showed a leftsided apical opacity, but both his sputum AFBs and culture were negative. His CD4 count was 39. FNA of the left neck mass was positive for $\mathrm{AFB}$, while the right one was negative. He was also found to have stool positive for AFB. The patient was started on RHZE.

Comment: In this case, the patient is US-born, aware of his HIV status, and appropriately treated, but his CD4 still puts him at high risk for disseminated disease. On exam he also exhibited a "cold abscess." Empiric treatment of a suspected 
abscess with antibiotics is appropriate, but respiratory isolation and workup for disseminated TB is also important.

Patient 8 is a 43 -year-old Mexican-American male who was brought to the ED by ambulance. He was found down on the street, unable to ambulate, and covered in feces. He was febrile, tachycardic, disheveled, cachectic, slightly confused but cooperative, and reported a productive cough and a history of HIV. He was incidentally found to have a left-sided supraclavicular mass. His mass was $5 \mathrm{~cm}$ across, soft, non-tender, non-pulsatile, and cyst like, with unclear margins. His chest X-ray showed bilateral pulmonary edema and a non-cavitary left-sided reticulonodular pattern. He was admitted to the medicine service, and his workup included a positive sputum and stool for AFB, while his ascites and CSF were both negative. FNA of his neck mass was AFB positive. The patient was started on RHZE and completed 5 months of treatment. His altered mental status was attributed to AIDS dementia.

Comment: This case illustrates that altered, possibly septic patients brought from the street could have disseminated TB and be contagious. Emergency department personel working in areas with a high prevalence of TB should consider respiratory isolation in these patients during the initial workup.

\section{Discussion}

Our case series does have some limitations. Indeed, the number of charts returned was less than half of the cases identified by diagnosis search, making our results less robust than anticipated. In addition, we may have retrieved fewer records than exist as a diagnosis of scrofula could have been omitted even though there was a positive culture. Perhaps a search by FNA result and culture would have been more sensitive. Nevertheless, some patterns do emerge, and they are in general agreement with previous findings.

When we examine the cases according to HIV status, we find a male predominance among HIV+ patients, while we find a balance among the sexes in HIV- patients, a pattern previously reported [5-7]. We found that half of all patients were HIV+, and all had AIDS by definition. Shriner reported a smaller proportion of HIV+ patients compared to HIV- in a very similar population, but 16 years earlier [7]. This may be a consequence of our uninsured indigent population that has not accessed available HIV therapy at a time when the incidence of HIV in the homeless, mentally ill, or drug-addicted population is increasing [8]. A decrease in immigration in Los Angeles has not been reported [9]. There is also a case report from Boston [10] published recently that exemplifies what will probably be a more common presentation of scrofula patients in the future: someone from a TB-endemic country who is also HIV positive. Case 4 in our series is a similar example.

The concerning issue with HIV+ patients who present with scrofula is that they are at much higher risk than immunocompetent patients of having disseminated disease and being contagious. In our series, most HIV+ patients had active pulmonary TB, while none of the HIV- patients did. Certainly, some HIV- patients will turn out to have pulmonary TB as well, but we were handicapped by the small number of cases. Shriner did report a $28 \%$ rate of abnormal CXR among HIV- patients and $90 \%$ of HIV+ patients; however, it is unclear if the abnormalities translated to active pulmonary TB or a latent disease [7].

Interestingly, lymph node characteristics did not appear different between the HIV- and the HIV+ population. That being said, the descriptions of the nodules in our charts were not as detailed as some other published reviews. For example, the location of the nodes was described as "cervical," but we don't know which specific chain was affected. Only rare charts had comments describing whether the node was matted or not. Furthermore, scrofula is classically described as a "cold abscess," yet only the charts of patients 2 and 7 specifically commented on whether there was palpable warmth or not. This is possibly secondary to the unclear nature of the diagnosis when the lesions were initially described and the lack of experience of the house staff providers in recognizing the characteristics of a tuberculous lymph node.

In general, in our series, diagnostic capabilities with AFB smears of fine-needle aspiration (FNA) samples appear good as six out of seven aspirates were positive. The one false negative had a culture that grew MTb. This is contrary to what has been generally reported in the literature. Shriner found $15 \% \mathrm{AFB}+$ lymph nodes by FNA in HIV- patients and $73 \%$ $\mathrm{AFB}+$ in $\mathrm{HIV}+$ patients. Levin found $47 \% \mathrm{AFB}+\mathrm{FNAs}$ in a population with unreported HIV status. Goel in 2001 found $62.82 \%$ FNA samples positive for AFB [11]. From these citations, it does appear that HIV status plays some role in the sensitivity of an FNA for AFB, but the extent the virus' influence is unclear. The differences in reported sensitivities could be due to our to our different mix of HIV+ and HIVpatients, our small sample size, or to chance. In addition, there is a selection bias since our retrospective review was based on the diagnosis of scrofula; if an FNA was negative despite the mass being caused by TB, the patient would not have been found in our search. Other explanations include differences in technique, laboratory experience, or other patient characteristics.

It was interesting to note that our patients were universally treated with pharmacologic therapy as recommended by the Centers for Disease Control (CDC) [12]. Only two patients ended up having an incision and drainage by the ENT service for unclear reasons. Earlier reports 
describe a much greater variability in the treatment of their patients [4]. The more recent literature is closer to our experience with a predominant use of 6 months of treatment with four drugs [6].

Having reviewed the characteristics of patients who have been treated at our institution for scrofula and compared our results to the current literature on the subject, we attempt recommendations for emergency physicians that will help them identify scrofula. We believe this is an important diagnosis not to miss as many of the patients with scrofula are at high risk of having pulmonary TB or laryngeal TB and thus are at high risk of being contagious.

First, one should look for a subacute presentation as all cases had a duration of a minimum of 2 weeks, which is in keeping with the general pathogenesis of tuberculosis. Second, identifying the patient's country of origin, regardless of the length of their stay in the US, is critical. If in doubt, the clinician can look up the distribution of TB on the CDC website (www.cdc.gov) or the World Health Organization (WHO) website (www.who.int). For US-born patients, one should investigate their HIV status, and any patient with a suggestive history should be considered high risk. Next, general TB constitutional symptoms should be sought: fever, night sweats, weight loss, and chronic cough. Finally, the emergency physician should examine the lymph node looking for the characteristics that define scrofula: large, usually painless swelling, sometimes erythematous, possibly matted with drainage. This information should give the clinician enough data to determine whether the patient is at a high risk for scrofula. If so, the patient should receive a chest $\mathrm{X}$-ray, be placed in isolation, and sputum AFBs should be obtained according to protocol to rule out active pulmonary disease.

\section{Conclusion}

The emergency physician has the tools to identify patients who present with a neck mass who are at high risk of having scrofula and disseminated TB. Prudent management of these patients would include admission to the hospital in respiratory isolation, baseline laboratory tests, chest X-ray, sputums for AFB, and a PPD in addition to an urgent fineneedle aspiration of the lesion.

\section{References}

1. Pratt et al. MMWR Weekly March 21, 2008/57(11);281-285 Trends in Tuberculosis - United States, 2007

2. Table E. Tuberculosis Cases by Year: Reporting Jurisdictions in California, 1998-2007 (pdf) in: California Department of Public Health http://www.cdph.ca.gov/programs/tb/Pages/WorldTBDayMarch24,2008.aspx, Accessed 23 Sept 2008
3. Clay J (2008), Scrofula, www.emedicine.com/ent/topic524.htm, Accessed 20 Sept 2008

4. Levin-Epstein AA, Lucente FE (1982) Scrofula-the dangerous masquerader. Laryngoscope 92(8 Pt 1):938-943

5. Ibekwe AO, Al Shareef Z, Al Kindy S, Diagnostic problems of tuberculous cervical adenitis (scrofula). Am J Otolaryngol 18(no. 3):202-205

6. Jha BC et al (2001) Cervical tuberculous lymphadenopathy: changing clinical pattern and concepts in management. Postgrad Med J 77(905):185-187

7. Shriner KA, Mathisen GE, Goetz MB (1992) Comparison of mycobacterial lymphadenitis among persons infected with human immunodeficiency virus and seronegative controls. Clinical Infectious Diseases: An Official Publication of the Infectious Diseases Society of America 15(4):601-605

8. Zolopa AR et al (1994) HIV and tuberculosis infection in San Francisco's homeless adults. Prevalence and risk factors in a representative sample. JAMA: The Journal of the American Medical Association 272(no. 6):455-461

9. Malone $\mathrm{N}$ et al. The foreign-born population: 2000, US Census 2000 brief, US Census Bureau (2003); available at: www.census. gov/prod/2003pubs/c2kbr-34.pdf Accessed 24 Sept 2008

10. Barnett K, Medzon R (2007) Scrofula as a presentation of tuberculosis and HIV. CJEM: Canadian Journal of Emergency Medical Care $=$ JCMU: Journal Canadien De Soins Médicaux D'urgence 9(3):176-179

11. Goel MM et al (2000) Polymerase chain reaction vs. conventional diagnosis in fine needle aspirates of tuberculous lymph nodes. Acta Cytologica 45(3):333-340

12. Blumberg HM et al (2003) American Thoracic Society/Centers for Disease Control and Prevention/Infectious Diseases Society of America: treatment of tuberculosis. Am J Respir Crit Care Med 167(4):603-662

Nicolas Forget is currently the Merkin Fellow of International Emergency Medicine at Los Angeles County-University of Southern California (LAC + USC) Hospital, a large urban public facility near downtown Los Angeles. He holds a B.Sc. from McGill University in Montreal, and his medical degree is from the University of Maryland at Baltimore. He completed his emergency medicine residency at LAC + USC. He is experienced in emergency medicine, trauma care, and international medicine, having worked in health education in Ecuador, observed rural medicine in Chile, followed an educational rotation in Cuba, participated in medical relief in Sri Lanka after the tsunami, and lectured in Liberia and Ghana while most recently working in Thailand with Burmese refugees.

Dr. Kathryn Challoner was born in Canada. She did her undergraduate studies at University of Toronto, Trinity College, then her MD at University of Ottawa. She completed two residencies: Family and Community Medicine at the Toronto General Hospital and Emergency Medicine at the LAC + USC Medical Center, Los Angeles, California. She also completed her MPH (Master of Public Health) at the Keck School of Medicine, University of Southern California. She is a Fellow of the American College of Emergency Medicine. Currently she is a member of Attending Staff in the Emergency Department of LAC + USC Medical Center, Co-Director of the International Division of Emergency Medicine, and an Associate Professor of Clinical Emergency Medicine at the Keck School of Medicine, University of Southern California. She developed international electives in West Africa for Keck School of Medicine students and served as team leader and symposium director on ten trips to Ghana and Liberia. She has also taught in Chile, on the Thailand-Burma border, and in Addis Ababa, Ethiopia. Her other educational interests include toxicology and correctional medicine. She is married with three children. 